\title{
Multimode Piezoelectric Shunt Damping With a Highly Resonant Impedance
}

\author{
S. O. R. Moheimani, Senior Member, IEEE, and S. Behrens
}

\begin{abstract}
This brief paper proposes a novel impedance structure for piezoelectric shunt damping. The impedance has a highly resonant nature and can be considered as a feedback controller applying a high gain at each resonance frequency of the base structure. Closed-loop stability of the system is proved and robustness properties of the associated controller are studied. Experimental results, demonstrating the effectiveness of the proposed procedure are presented.
\end{abstract}

Index Terms-Active control, feedback, flexible structures, piezoelectric, vibration control.

\section{INTRODUCTION}

$\mathbf{P}$ IEZOELECTRIC materials are being increasingly used in vibration control applications. In particular, they are quite useful in active control [1]-[4] and passive control [5], [6] of linearly elastic structures. The latter is also referred to as shunt damping [7]. In active vibration control applications, a feedback controller is designed and implemented using high-voltage amplifiers capable of driving highly capacitive loads. Stability is an important issue in such designs as the spillover effect due to ignored out-of-bandwidth modes may destabilize the closed loop system [8], [9].

The passive control technique, as proposed in [5] acts on a single mode of the base structure and requires a simple RL shunt circuit. The shunt circuit, along with the capacitance of the piezoelectric transducer forms a RLC circuit that is tuned to a specific resonance frequency of the vibrating structure, resulting in vibration reduction for that specific mode. An attractive feature of this method is that stability of the resulting electromechanical system is guaranteed. Furthermore, the shunt circuit can be implemented using a resistor and an inductor.

Often, several vibration modes of a structure are to be suppressed. There has been a number of attempts at extending the method of [5] to allow for multi-mode vibration reduction. References [7], [10] address this problem by introducing current blocking circuits inside each R-L branch. The difficulty associated with this technique, however, is that the size of the shunting circuit increases rapidly as the number of modes that are to be shunt damped is increased. Reference [11] suggests a more practical shunt circuit for multiple mode piezoelectric shunt damping by introducing current-flowing circuits in each branch. An alternative multi-mode shunt damping circuit was suggested by Hollkamp [12]. Despite the author's conjectures

Manuscript received September 30, 2002; revised March 23, 2003. Manuscript received in final form June 3, 2003. Recommended by Associate Editor F. Svaricek. This work was supported by the Australian Research Council.

The authors are with the School of Electrical Engineering and Computer Science, University of Newcastle, NSW, Australia (e-mail: reza@ee.newcastle.edu.au).

Digital Object Identifier 10.1109/TCST.2004.824318 on the effectiveness of this circuit, no straightforward method for determining the circuit components is proposed.

One of the major difficulties that often arises in implementing these shunt impedances is the fact that one may need to have access to rather large inductors if the low frequency modes are to be shunt damped. The mainstream approach to addressing this problem appears to be centered around electronic implementation of these inductors using Gyrator circuits [13]. This may be a reasonable proposition if a very small number of modes are to be shunt damped. However, for a large number of modes a more practical solution is needed.

Reference [14] proposes an effective means of digital implementation of an impedance circuit for piezoelectric shunt damping. This circuit allows one to implement any admittance transfer function, as long as its transfer function is stable, and at least proper. Indeed the circuit may be used to implement passive/active shunts, as opposed to purely passive shunts. For an overview of current active/passive techniques, the reader is referred to [15].

This paper proposes that the problem of passive control of vibration can be considered as a feedback control problem with a very specific feedback structure. This leads to a unifying approach to vibration control since the passive and semi-active [15] vibration control methodologies can now be cast into the same framework.

The remainder of the paper continues as follows. Section II is concerned with the piezoelectric shunt damping problem and the specific feedback structure associated with it. Section III introduces a class of stabilizing shunt controllers that can be effective in reducing vibrations of the base structure. Section IV studies properties of the proposed impedance structure. Section V includes our experimental results, and Section VI concludes the overall of the paper.

\section{Piezoelectric Shunt Damping}

Consider the system depicted in Fig. 1 in which a piezoelectric transducer is attached to the surface of a flexible structure using strong adhesive material. The piezoelectric transducer is shunted by an electrical impedance, $Z$. The vector $P$ signifies the direction of polarization vector of the piezoelectric material. As the structure deforms, possibly due to a disturbance $w$, an electric charge distribution develops inside the piezoelectric crystal. The transducer can be thought of as a parallel plate capacitor. The electric charge will result in a voltage difference, $v$ across the conducting surfaces of the piezoelectric transducer which in turn causes the flow of electric current, $i$ through the impedance. This causes a loss of energy. Hence, the electric impedance may be viewed as a means of extracting mechanical energy from the base structure via the piezoelectric transducer. 


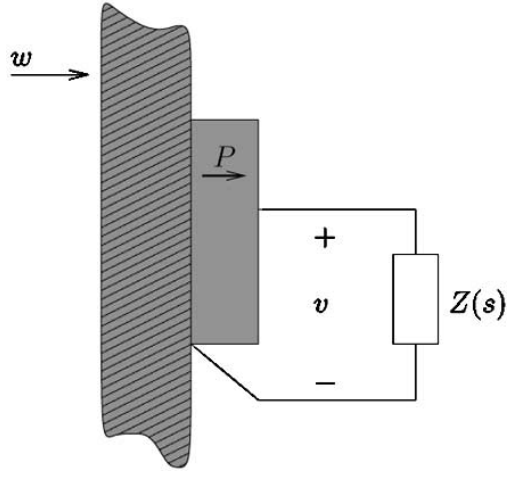

Fig. 1. A piezoelectric laminate shunted to an impedance $Z(s)$.

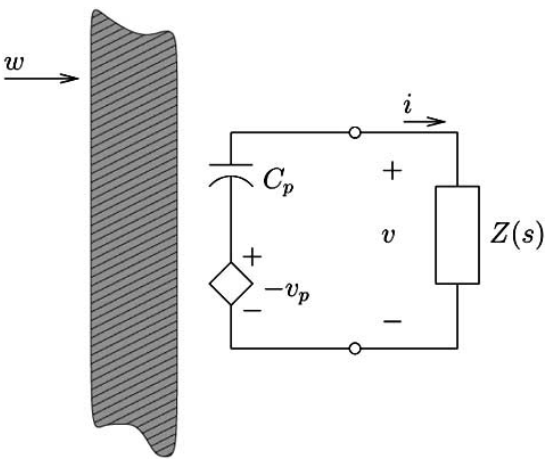

Fig. 2. Electrical equivalent of the system in Fig. 1.

Fig. 2 depicts the electrical equivalent of the piezoelectric transducer [16]. If the impedance is removed from the circuit, i.e., if the piezoelectric transducer is left open circuited, then the voltage measured across the conducting terminals of the piezoelectric transducer is equivalent to $v_{p}$. This voltage is entirely due to the disturbances acting on the structure and/or nonzero initial conditions and is an indication of the mechanical strain inside the base structure. It should be clear that as long as the base structure is not at rest, $v_{p}$ may be nonzero. To this end, it is assumed that $v_{p}$ is related to $w$ via a transfer function $G_{v w}$. That is

$$
v_{p}(s)=G_{v w}(s) w(s) .
$$

Now, one may assume that there are no disturbances acting on the structure while a voltage source, $v$ is attached across the conducting terminals of the piezoelectric transducer. In this case, the voltage $v_{p}$ is entirely due to $v$ and is related to it via a transfer function $G_{v v}$. That is

$$
v_{p}(s)=G_{v v}(s) v(s), \quad w(s)=0 .
$$

The transfer function $G_{v v}$ may be written in the general form [4],

$$
G_{v v}(s)=\sum_{i=1}^{M} \frac{\gamma_{i}}{s^{2}+2 \zeta_{i} \omega_{i} s+\omega_{i}^{2}}
$$

where

$$
\gamma_{i} \geq 0 \quad \text { for } i=1,2, \ldots
$$

If the modal analysis procedure is used in developing a model of the form (3), one should expect $M \rightarrow \infty$. However, assuming a very large number for $M$ is quite acceptable, and more realistic than $M \rightarrow \infty$, e.g., see [17].

If the piezoelectric transducer is attached to the structure such that the vector $P$ is pointing to the opposite direction, a negative sign should be added to (3). If the base structure is disturbed by $w$ and a voltage $v$ is simultaneously applied across the terminals of the piezoelectric transducer then due to the linearity of the system we may write

$$
v_{p}(s)=G_{v w}(s) w(s)+G_{v v}(s) v(s) .
$$

From (4) it can be understood that while the disturbance $w$ is disturbing the base structure, the voltage $v(s)$ applied across the piezoelectric terminals may be used to reduce the effect of this unwanted disturbance. In a typical feedback control problem, a sensor is used to measure a property of the structure for feedback. This may be the acceleration at some point, as measured by an accelerometer, or even the voltage measured at the open terminals of another piezoelectric transducer attached to the structure at a different point.

Shunting the piezoelectric transducer with the impedance $Z$, as in Fig. 2 removes the need for an additional sensor. This, however, is achieved at the expense of having to deal with a more complicated feedback control problem. This point will be further clarified in the next section.

To visualize the underlying feedback control structure, we need to identify a number of variables such as the control signal, the measurement, the disturbance and the physical variable that is to be regulated.

The underlying feedback structure can be identified by noticing that the current may be written as

$$
i(s)=-\left(v_{p}(s)+v(s)\right) C_{p} s .
$$

Furthermore

$$
v(s)=Z(s) i(s) .
$$

Equations (4)-(6) suggest the feedback structure depicted in Fig. 3. Fig. 3 represents a rather complicated feedback structure as the controller, $Z(s)$ is itself inside an inner feedback loop.

In the following sections, a number of issues relevant to this system will be studied. The most important point that will be addressed is the problem of stability of the closed-loop system in Fig. 3. This issue will be addressed for the equivalent system depicted in Fig. 4, as it is more straightforward to study the closed-loop stability of the underlying system with $Y(s)=$ $(1 / Z(s))$ as the controller.

\section{A Class of Stabilizing Shunts}

In the previous section it was demonstrated that the problem of piezoelectric shunt damping was equivalent to a feedback control problem, in which the impedance shunted to the piezoelectric transducer can be considered as the controller. This observation has interesting implications as it enables one to employ systems theoretic tools in designing effective impedances for piezoelectric shunt damping systems. 


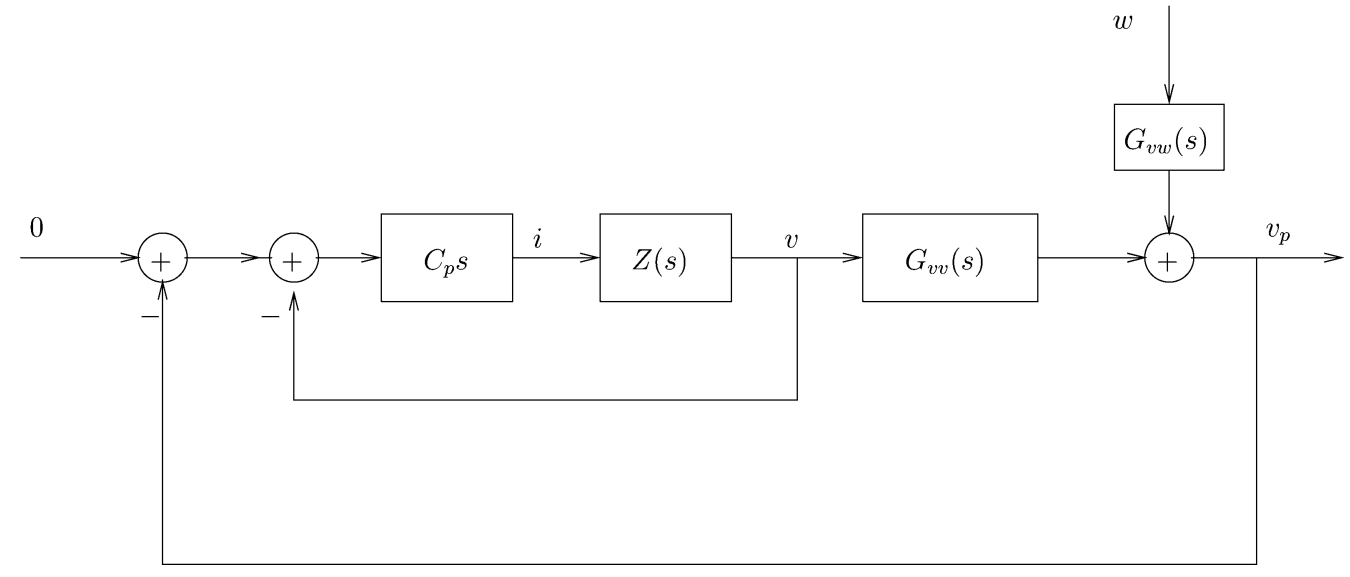

Fig. 3. The feedback structure associated with the shunt damping problem in Fig. 2.

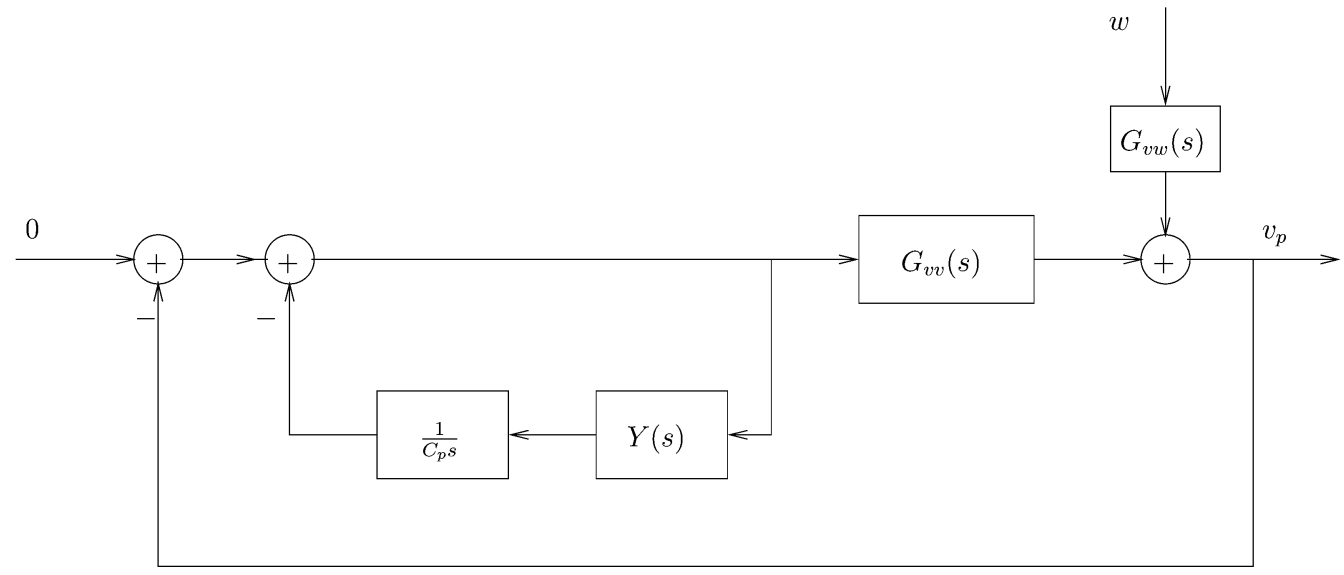

Fig. 4. Equivalent circuit for the system in Fig. 3 replacing $Z$ with $Y$.

Let us consider the feedback problem associated with the system in Fig. 4. It represents a feedback loop described by

$$
T_{v_{p} w}(s)=\frac{G_{v w}(s)}{1+K(s) G_{v v}(s)}
$$

where the real controller $Y(s)$ is itself inside an inner feedback loop; i.e.,

$$
K(s)=\frac{1}{1+\frac{Y}{C_{p} s}} .
$$

To this end, we wish to determine a parameterization of all admittance transfer functions, $Y(s)$ that renders the inner feedback loop internally stable. Youla parameterization [18] of all stabilizing controllers for a stable plant can be used for this purpose. Once such a parameterization is determined, we will proceed to finding a specific admittance that guarantees closed loop stability of the overall system. It should be pointed out that internal stability of the inner feedback loop is indeed important since it represents the interactions between the shunted admittance and the capacitive component of the piezoelectric transducer. By ensuring that this loop is internally stable we can guarantee that problems such as unstable pole zero cancellation will not occur.
Youla parameterization of all stabilizing controllers $\tilde{K}$ for a given stable plant $\tilde{G}(s)$ can be written as

$$
\tilde{K}(s)=\frac{Q(s)}{1-Q(s) \tilde{G}(s)}
$$

where $Q(s)$ can be any stable transfer function (not necessarily proper). However, it should be noted that for the inner feedback loop in Fig. 4 the plant is an integrator, i.e., $\tilde{G}(s)=\left(1 / C_{p} s\right)$ and hence unstable. If the plant $\tilde{G}(s)$ has unstable poles instead of using Youla parameterization for an unstable plant [18], we may use the above parameterization, as long as $Q(s)$ satisfies the following conditions (see [19, Lemma 15.4]):

1) $Q(s)$ must be stable and proper;

2) $Q(s)$ must have zeros at the unstable poles of $\tilde{G}(s)$;

3) $1-Q(s) \tilde{G}(s)$ must have zeros at the unstable poles of $\tilde{G}(s)$.

Noting that $\tilde{G}(s)=1 /\left(C_{p} s\right)$, for the inner feedback loop, all these conditions can be enforced as long as $Q(s)$ is chosen as

$$
Q(s)=H(s) C_{p} s
$$

with $H(s)$ satisfying the following conditions:

1) $H(s)$ is stable and strictly proper;

2) $1-H(s)$ has a zero at the origin. 


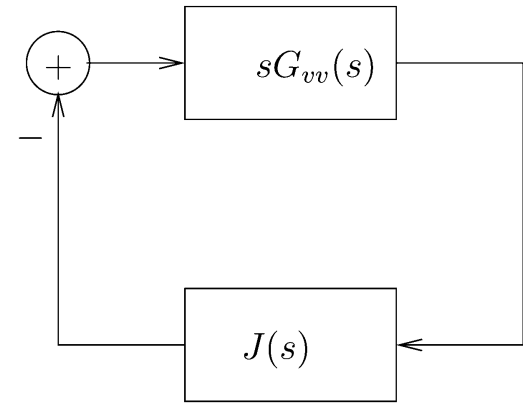

Fig. 5. Equivalent system for study of closed-loop stability.

Therefore, any admittance chosen as

$$
Y(s)=\frac{H(s)}{1-H(s)} C_{p} s
$$

with $H(s)$ satisfying the above two conditions will guarantee internal stability of the inner feedback loop resulting in the effective controller

$$
K(s)=1-H(s)
$$

which is stable. Furthermore, if $H(s)$ is chosen to be

$$
H(s)=1-s J(s)
$$

with $J(s)$ a strictly positive real system, the closed loop stability of the overall system can be guaranteed. This can be verified by observing that stability of the overall system is equivalent to the stability of the negative feedback loop in Fig. 5.

It can be verified that $s G_{v v}(s)$ is a positive real system [20]. Therefore, if $J(s)$ is chosen to be a strictly positive real system, closed loop stability of the overall system will be guaranteed [21], [20].

Purpose of the shunted admittance is to add damping to the structure within a specific bandwidth. This amounts to moving closed-loop poles of the system deeper into the left half of the complex plane. Given the highly resonant nature of the base structure, as evident from (3), one way of achieving this goal would be to choose a highly resonant structure for the shunted impedance. An impedance structure that results in favorable closed loop performance can be constructed by choosing the following form for $J(s)$ :

$$
J(s)=\sum_{i=1}^{N} \frac{\alpha_{i} s}{s^{2}+2 d_{i} \omega_{i} s+\omega_{i}^{2}}
$$

where $\alpha_{i} \geq 0$ for $i=1,2, \ldots, N$ and

$$
\sum_{i=1}^{N} \alpha_{i}=1
$$

This choice for $J(s)$ results in the effective controller

$$
K(s)=\sum_{i=1}^{N} \frac{\alpha_{i} s^{2}}{s^{2}+2 d_{i} \omega_{i} s+\omega_{i}^{2}} .
$$

Also, the admittance transfer function that has to be implemented is

$$
Y(s)=\frac{\sum_{i=1}^{N} \frac{\alpha_{i}\left(2 d_{i} \omega_{i} s+\omega_{i}^{2}\right)}{s^{2}+2 d_{i} \omega_{i} s+\omega_{i}^{2}}}{1-\sum_{i=1}^{N} \frac{\alpha_{i}\left(2 d_{i} \omega_{i} s+\omega_{i}^{2}\right)}{s^{2}+2 d_{i} \omega_{i} s+\omega_{i}^{2}}} C_{p} s
$$

To this end, it needs to be pointed out that the transfer function in (11) is not a minimal system. A minimal realization of (11) is discussed in the next section when we attempt to study properties of the shunting admittance.

A distinct characteristic of the admittance (11) is its inherent stabilizing properties. The existence of the out-of-bandwidth modes in (3) has the potential to cause closed loop instabilities as a result of the spill-over effect [8], [9]. However, due to the specific structure of $Y(s)$, the closed loop stability in the presence of out-of-bandwidth modes is guaranteed. A further interesting property of $Y(s)$ is its robustness with respect to inaccurate in-bandwidth model of the base structure. If $Y(s)$ is designed based on a model (3) with inaccurate parameters, $\omega_{i}, \zeta_{i}$, and $\alpha_{i}$, the closed loop system will still remain stable, although the closed loop performance may significantly deteriorate.

\section{Properties of THE PRoposed IMPEDANCE}

In this section, we concentrate on the impedance of the shunting element, i.e., $Z(s)=(1 / Y(s))$. It can be verified that $Z(s)$ is a strictly positive real transfer function; i.e., it is stable and $\operatorname{Re}\{Z(j \omega)\}>0$ for all $\omega \in \mathbf{R}$. Therefore, $Z(s)$ must be implementable using passive circuit elements; i.e., resistors, inductors and capacitors. To synthesize a network of passive elements for $Z(s)$, one has to start with the representation of the shunt impedance given in (12) at the bottom of the page.

A number of synthesis techniques can be used to implement such an impedance (see, e.g., [22], [23]). However, it is not clear how a passive network should be extracted from the above representation of the shunt impedance as standard synthesis techniques result in circuits that require elements such as gyrators. Nevertheless, if such a synthesis is found it is very likely that it would be unrealizable due to the size of the necessary inductors. In a typical vibration control problem, one is often interested in suppressing low-frequency modes. Therefore, one should expect to need large inductors for this purpose. Inductors of the order of several thousand henries are not uncommon in such applications. The synthetic impedance circuit suggested in [14] is an effective method for digital implementation of a given impedance transfer function (see also [24]). It is instructive to consider the situation where only one mode is to be shunt damped. This can be achieved by setting the admittance $Y(s)$ in (11) equal to the $i$ th term. That is

$$
Z(s)=\frac{s / C_{p}}{2 d_{i} \omega_{i} s+\omega_{i}^{2}} .
$$

$$
Z(s)=\frac{\sum_{i=1}^{N} \alpha_{i} s \prod_{\ell=1, \ell \neq i}^{N}\left(s^{2}+2 d_{i} \omega_{i} s+\omega_{i}^{2}\right)}{C_{p} \sum_{i=1}^{N} \alpha_{i}\left(2 d_{i} \omega_{i} s+\omega_{i}^{2}\right) \prod_{\ell=1, \ell \neq i}^{N}\left(s^{2}+2 d_{i} \omega_{i} s+\omega_{i}^{2}\right)} .
$$




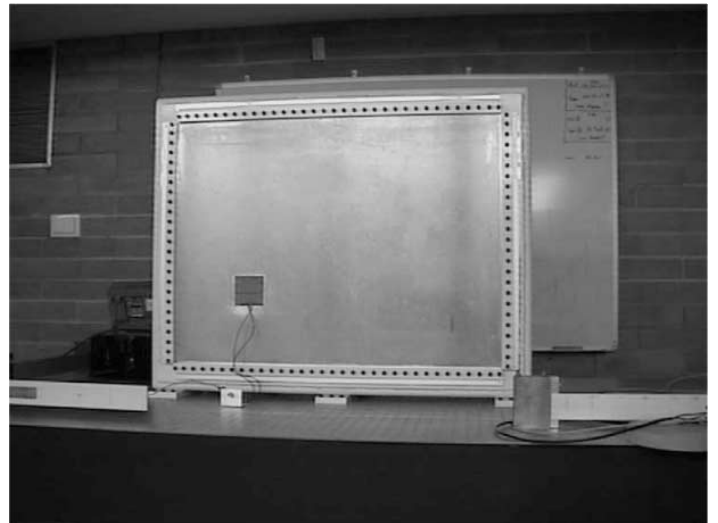

Fig. 6. Piezoelectric laminated plate structure.

This is equivalent to the parallel connection of a resistor

$$
R=\frac{1}{2 d_{i} \omega_{i} C_{p}}
$$

with an inductor

$$
L=\frac{1}{\omega_{i}^{2} C_{p}} .
$$

The parallel RL circuit for single mode piezoelectric shunt damping was proposed in [25], in which a similar choice for $L$ is proposed. Extension to multimode systems is also investigated by the same author [7]. However, the methodology proposed in the reference is considerably more complicated, and far less effective, than that proposed here.

\section{EXPERIMENTAL RESULTS}

The proposed control scheme was validated experimentally on a piezoelectric laminate plate structure. A photograph of the structure in question is shown in Fig. 6. Two piezoelectric patches were bonded to either sides of the structure, in a collocated configuration, using a strong adhesive material. One of the piezoelectric patches was used as an actuator to generate a disturbance while the other patch was used as a shunting layer (see Fig. 7). For a detailed description of the experimental apparatus, the reader is referred to [26], [27].

From Fig. 7 it can be observed that the disturbance is the voltage applied to an identical piezoelectric actuator collocated with the shunted piezoelectric patch. Since the two piezoelectric transducers are identical, and considering the orientation of polarization vectors of the two transducers, we may write

$$
G_{v w}(s)=-G_{v v}(s) .
$$

Therefore, the block diagram in Fig. 4 can be reduced to that shown in Fig. 8. An advantage of using this configuration is that by measuring unshunted $G_{v w}$, the collocated transfer function $G_{v v}$ can be automatically determined. Otherwise, if a collocated piezoelectric transducer is not available, $G_{v v}$ can be determined by applying a voltage to the transducer, measuring the current that flows as a result of it, and from the knowledge of $C_{p}$.

A more detailed schematic of the experimental setup is plotted in Fig. 9. The disturbance is applied to one of the piezoelectric patches using a voltage source capable of driving highly capacitive loads. The admittance transfer function is

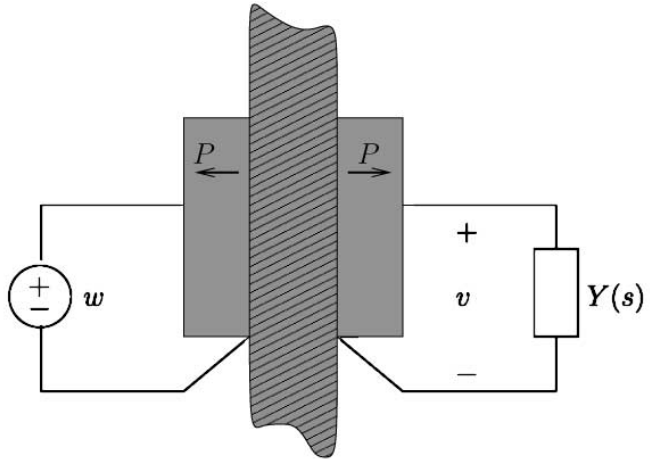

(a)

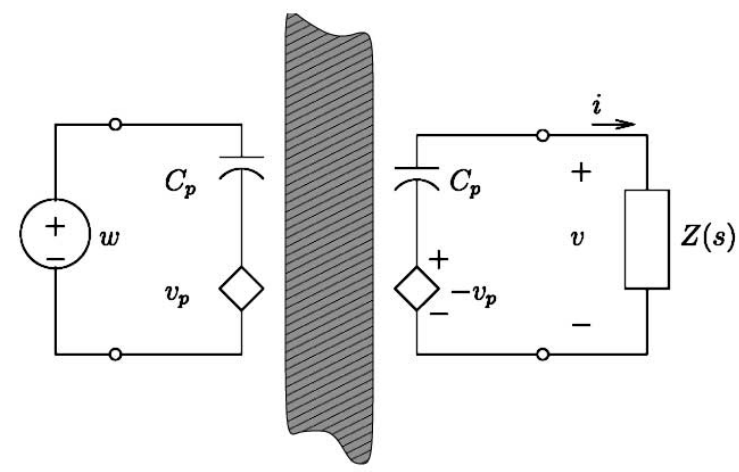

(b)

Fig. 7. (a) Structure with collocated piezoelectric transducers and (b) its electrical equivalent.

implemented digitally using a current controlled voltage source. The voltage across the shunting piezoelectric layer is measured and a current $i=Y v_{s}$ is supplied. The admittance transfer function $Y(s)$ is implemented digitally using a dSPACE DS1103 rapid prototyping system. For a detailed description of the synthetic admittance networks the reader is referred to [24], [14].

In order to design an effective shunt, it is necessary to obtain analytical models for $G_{v v}$ and $G_{x w}$, the transfer function from the disturbance voltage to the displacement at a point on the surface as measured by a laser vibrometer. For majority of systems obtaining analytical models may be too difficult, so a system identification method may have to be employed. Subspace based system identification techniques have proved to be efficient means of identifying dynamics of high order highly resonant systems (see [28], [29] and references therein).

Using a Polytec laser scanning vibrometer (PSV-300) and a Hewlett Packard spectrum analyzer (35670A), the frequency responses for $G_{v v}$ and $G_{x w}$ were obtained. The measurements were carried out in open loop, i.e., the terminals of the shunting layer were left open when $G_{x w}$ was being measured while they were connected to the spectrum analyzer when $G_{v v}$ was being measured. Using the already mentioned system identification technique, $G_{v v}$ and $G_{x w}$ were identified over the frequency range of 30-250 Hz. The models include the first 6 modes of the plate. Frequency responses of the identified transfer functions are shown in Fig. 10. The first, second, third, fifth, and sixth structural modes were chosen for control due to their highly resonant nature. 


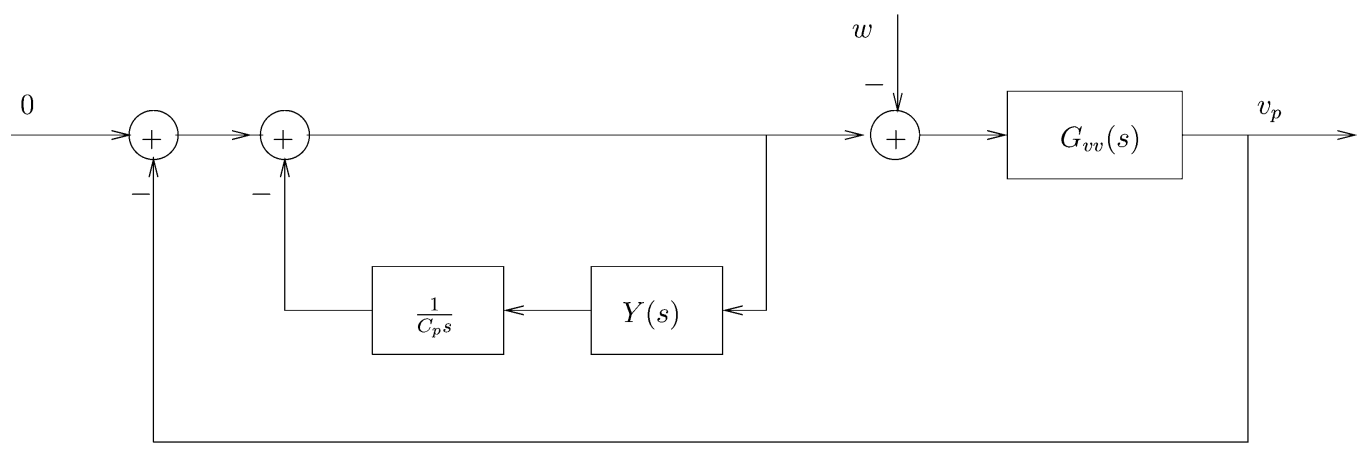

Fig. 8. The feedback structure with the disturbance applied to the collocated piezoelectric transducer.

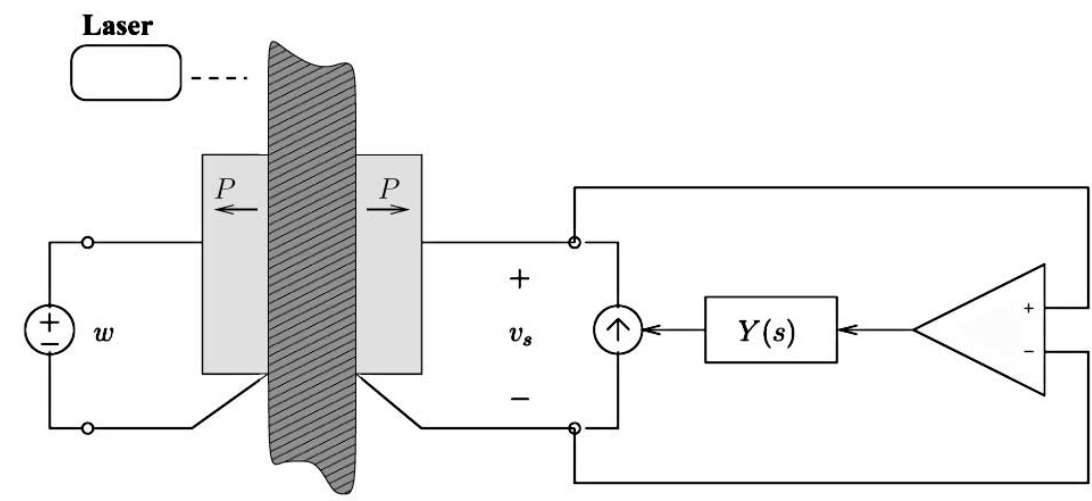

Fig. 9. Experimental set up.
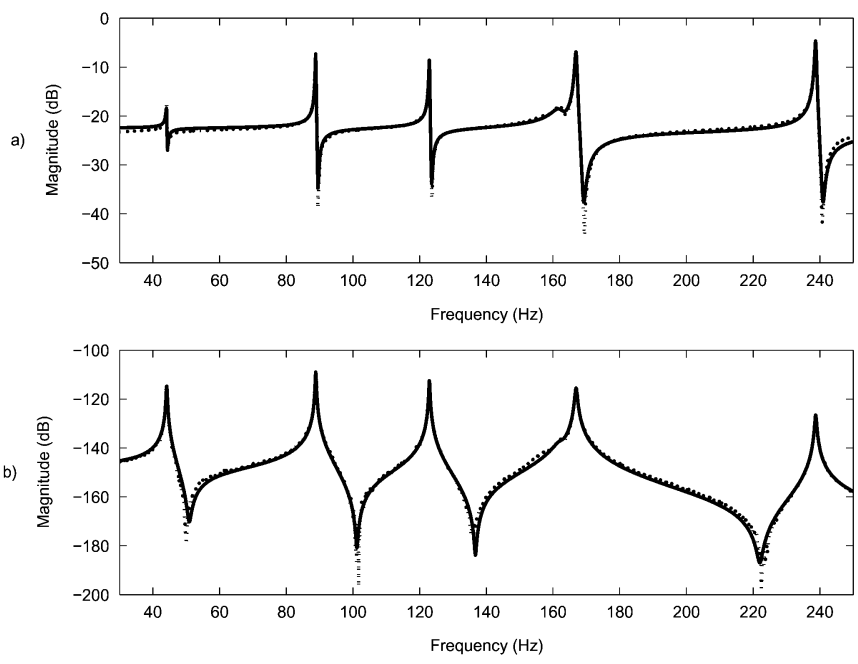

Fig. 10. Frequency response (a) $\left|G_{v v}(s)\right|(\mathrm{v} / \mathrm{v})$ and (b) $\left|G_{x w}(\mathbf{x}, s)\right|(\mathrm{m} / \mathrm{v})$, for the piezoelectric laminated plate structure. Experimental data $(\cdots)$ and model obtained using subspace based system identification (-).

An admittance of the form proposed in (11) was then designed. In order to achieve good performance, appropriate values for the damping parameters $\alpha_{1}, \ldots, \alpha_{6}$ and $d_{1}, \ldots, d_{6}$ needed to be determined. To simplify the desing, parameters $\alpha_{1}, \ldots, \alpha_{3}$, and $\alpha_{5}$ and $\alpha_{6}$ were set equal to $1 / 5$ while $\alpha_{4}$ was fixed at 0 , as its associated mode was to be left uncontrolled. This ensured that condition (10) was satisfied. The piezoelectric capacitance, $C_{p}$ was experimentally determined to be $67.9 \mathrm{nF}$.
Furthermore, the first six resonance frequencies of the structure, $\Omega=\left\{\omega_{1}, \omega_{2}, \ldots, \omega_{6}\right\}$ were extracted from the identified models. The following optimization problem was then solved:

$$
D^{*}=\arg \min _{D>0}\left\|T_{x w}\right\|_{2}
$$

where $D^{*}=\left\{d_{1}^{*}, d_{2}^{*}, \ldots, d_{6}^{*}\right\}$, and $T_{x w}$ represents the shunted transfer function from the disturbance voltage $w$ to the displacement $x$ as measured by a vibrometer. The purpose of the optimization is to minimize the $H_{2}$ norm of $T_{x w}$. This will subsequently result in adding extra damping to the structure by shifting closed loop poles of the system deeper into the left half of the complex plane. The optimization problem was solved for a number of initial guesses, and a solution was found; $d_{1}^{*}=$ $0.0049, d_{2}^{*}=0.0076, d_{3}^{*}=0.0063, d_{5}^{*}=0.0087$, and $d_{6}^{*}=$ 0.0080 . Notice that the fourth mode is left uncontrolled. The reason for this will be clarified soon.

Using the values $D^{*}, \Omega$, and $C_{p}$ we can sketch the Nyquist diagram for the impedance $Z(s)$. This is shown in Fig. 11. From the Nyquist diagram we can see that the impedance is strictly positive real [22], that is $Z(s)$ is stable and $\operatorname{Re}[Z(j \omega)]>0$ : $\forall \omega \in \mathbf{R}$. Therefore, guaranteeing that the impedance is realizable using passive circuit elements. Such a realization using resistors, capacitors and inductors may be impossible as the size of the required inductive elements may be too large. Hence, the need for digital implementation of the impedance.

Simulations of the closed loop response, as shown in Fig. 12, demonstrate that the first, second, third, fifth, and sixth resonant 


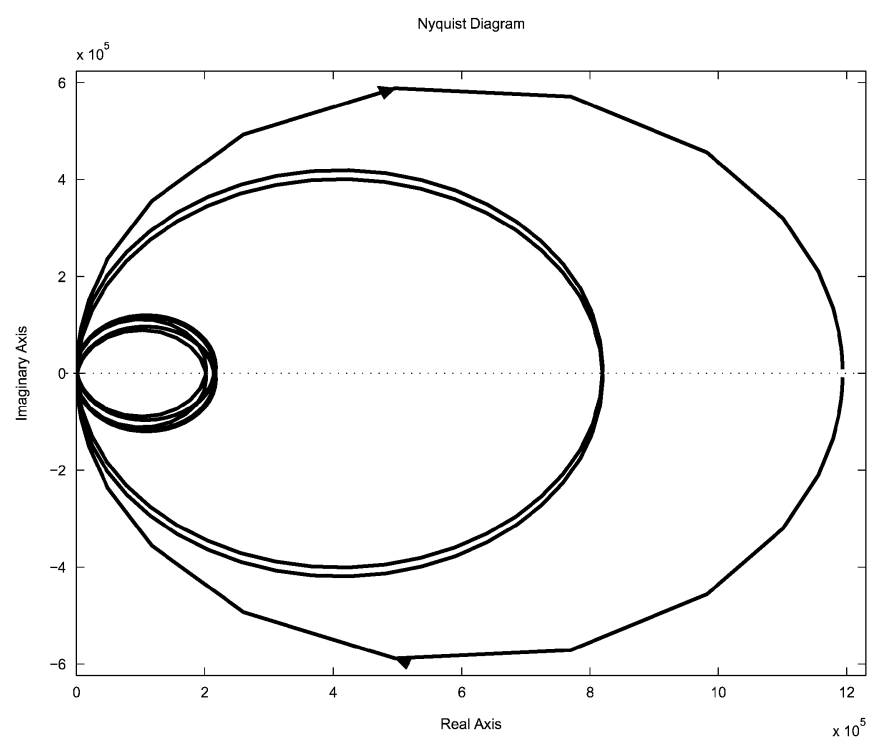

Fig. 11. Nyquist plot for impedance $Z(s)$.

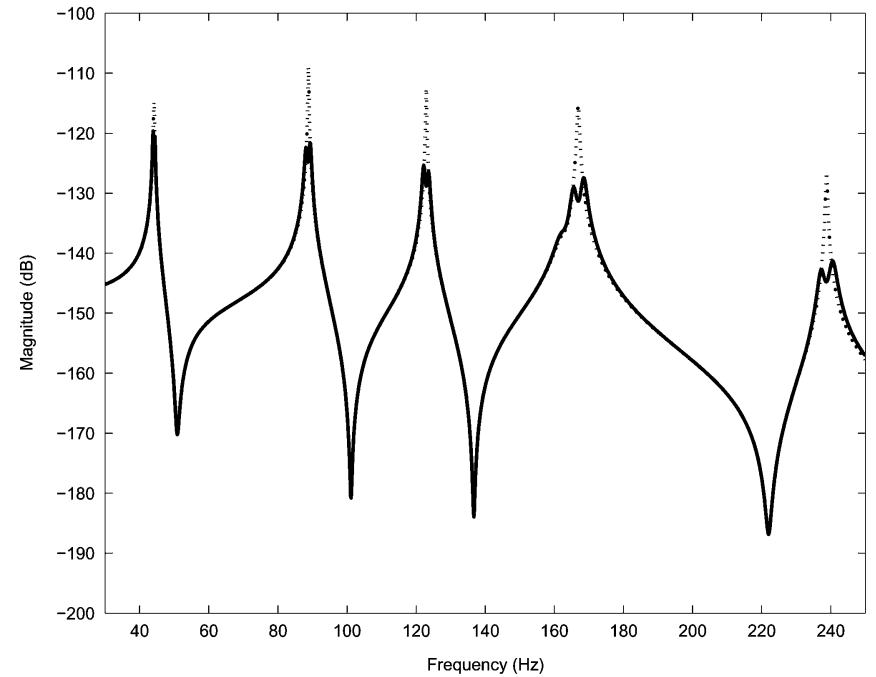

Fig. 12. Simulated open-loop (..$)$ versus closed-loop response $(-)$.

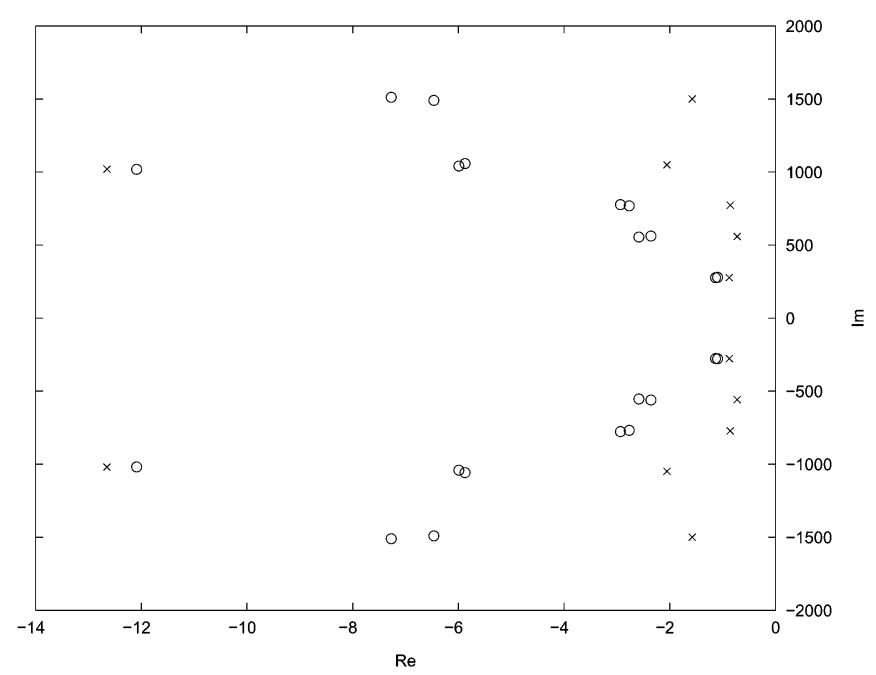

Fig. 13. Simulated open-loop $(x)$ and closed-loop $(o)$ poles.

amplitudes are expected to decrease by $5.0,12.6,12.7,11.8$, and $14.7 \mathrm{~dB}$, respectively. Fig. 13 shows the locations of the closed-loop and open-loop poles. We can see from Fig. 13, that

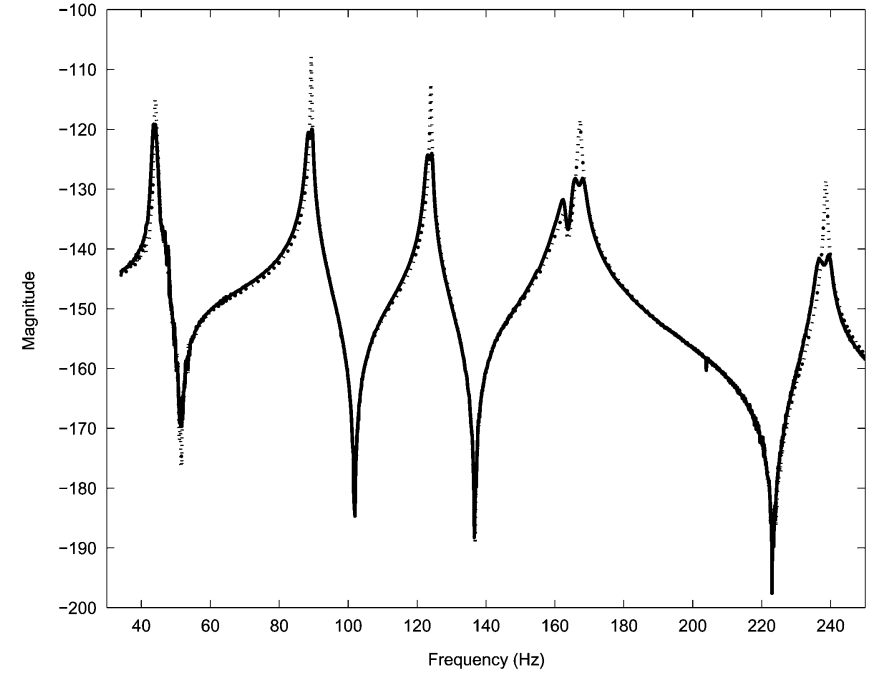

Fig. 14. Experiential open-loop $(\cdots)$ versus closed-loop response $(-)$.

the closed loop poles have been pushed deeper into the left-half of the complex plane. By shifting the poles to the left, effectively, more damping has been added to the structure. Note that the fourth mode had sufficient damping compared to the other undamped structural modes. Hence, it was left uncontrolled.

The proposed admittance $Y(s)$ was then digitally implemented using the synthetic admittance circuit [24], [14] on the piezoelectric laminated plate. A comparison of the open loop and closed loop (unshunted and shunted) experimental results are shown in Fig. 14. It can be observed that the proposed shunt is very effective in reducing the resonant amplitudes for the first, second, third, fifth, and sixth modes. However, the shunt's performance for the first mode is very limited. This can be attributed to the location at which the piezoelectric transducers were placed on the plate structure. Resonant amplitudes were successfully reduced by $4.2,12.0,11.5,10.1$, and $12.6 \mathrm{~dB}$.

\section{CONCLUSION}

This brief paper demonstrated that the problem of vibration control with a shunted piezoelectric actuator is a feedback control problem. Moreover, we determined a parameterization of stabilizing shunt impedances and proposed a specific shunt impedance with good vibration suppressing properties. Effectiveness of the proposed impedance was demonstrated experimentally.

\section{REFERENCES}

[1] E. F. Crawley and J. de Luis, "Use of piezoelectric actuators as elements of intelligent structures," AIAA J., pp. 1373-1385, Oct. 1987.

[2] D. J. Inman and E. Garcia, "Smart structures for vibration suppression," in Proc. Fourth International Conference on Recent Advances in Structural Dynamics, Southampton, U.K., July 15-18, 1991.

[3] A. Baz and S. Poh, "Performance of an active control system with piezoelectric actuators," J. Sound Vibr., vol. 126, no. 2, pp. 327-343, 1988.

[4] D. Halim and S. O. R. Moheimani, "Spatial resonant control of flexible structures-Application to a piezoelectric laminate beam," IEEE Trans. Contr. Syst. Technol., vol. 9, pp. 37-53, Jan. 2001.

[5] N. W. Hagood and A. von Flotow, "Damping of structural vibrations with piezoelectric materials and passive electrical networks," J. Sound Vibr., vol. 146, no. 2, pp. 243-268, 1991.

[6] S. Behrens, "Passive and Semi-Active Vibration Control of Piezoelectric Laminates," Master's Thesis, Dep. Elect. Comput. Eng., Univ. Newcastle, 2000. 
[7] S. Y. Wu, "Method for multiple mode shunt damping of structural vibration using a single PZT transducer," in Proc. SPIE Symposium on Smart Structures and Materials-Smart Structures and Intelligent Systems, Mar. 1998, pp. 159-167.

[8] M. J. Balas, "Active control of flexible systems," J. Optimiz. Theory Applicat., vol. 25, no. 3, pp. 415-436, 1978.

[9] - "Feedback control of flexible systems," IEEE Trans. Automat. Contr., vol. AC-23, Apr. 1978.

[10] S. Y. Wu, "Piezoelectric Shunts for Simultaneous Vibration Reduction and Damping of Multiple Vibration Modes,", July 1998.

[11] S. Behrens, S. O. R. Moheimani, and A. J. Fleming, "Multiple mode current flowing passive piezoelectric shunt controller," J. Sound Vibr., vol. 266, no. 5, pp. 929-942, Oct. 2003.

[12] J. J. Hollkamp, "Multimodal passive vibration suppression with piezoelectric materials and resonant shunts," J. Intell. Mater. Syst. Struct., vol. 5, Jan. 1994.

[13] R. H. S. Riodan, "Simulated inductors using differential amplifiers," Electron. Lett., vol. 3, no. 2, pp. 50-51, 1967.

[14] A. J. Fleming, S. Behrens, and S. O. R. Moheimani, "Synthetic impedance for implementation of piezoelectric shunt damping circuits," Electron. Lett., vol. 36, no. 18, pp. 1525-1526, 2000.

[15] J. Tang and K. W. Wang, "Active-passive hybrid piezoelectric networks for vibration control: comparisons and improvement," Smart Mater. Struct., vol. 10, no. 4, pp. 794-806, 2001.

[16] J. J. Dosch, D. J. Inman, and E. Garcia, "A self-sensing piezoelectric actuator for collocated control," J. Intell. Mater. Struct., vol. 3, no. 1, pp. 166-185, 1992.

[17] P. C. Hughes, "Space structure vibration modes: How many exist? Which ones are important?," IEEE Contr. Syst. Mag., pp. 22-28, Feb. 1987.

[18] M. Vidyasagar, Control System Synthesis, A Factorization Approach. Cambridge: MIT Press, 1987.
[19] G. C. Goodwin, S. F. Graebe, and M. E. Salgado, Control System Design: Prentice Hall, 2001.

[20] H. K. Khalil, Nonlinear Systems, 2nd ed. Upper Saddle River, NJ: Prentice-Hall, 1996.

[21] S. M. Joshi and S. Gupta, "On a clss of marginally stable positive-real systems," IEEE Trans. Automat. Contr., vol. 41, no. 1, pp. 152-155, Jan. 1996.

[22] J. E. Storer, Passive Network Synthesis. New York: McGraw-Hill, 1957.

[23] B. D. O. Anderson and S. Vongpanitlerd, Network Analysis and Synthesis. Englewood Cliffs, NJ: Prentice Hall, 1973.

[24] A. J. Fleming, S. Behrens, and S. O. R. Moheimani, "Optimization and implementation of multi-mode piezoelectric shunt damping systems," Trans. Mechatron., vol. 7, no. 1, pp. 87-94, Mar. 2002.

[25] S. Y. Wu, "Piezoelectric shunts with parallel R-L circuit for smart structural damping and vibration control," in Proc. SPIE Symposium on Smart Structures and Materials-Passive Damping and Isolation, Mar. 1996, pp. 259-269.

[26] D. Halim and S. O. R. Moheimani, "An optimization approach to optimal placement of collocated piezoelectric actuators and sensors on a thin plate," Mechatronics, vol. 13, no. 1, pp. 27-47, Feb. 2003.

[27] S. Behrens, A. J. Fleming, and S. O. R. Moheimani, "A broadband controller for shunt piezoelectric damping of structural vibration," Smart Mater. Struct., vol. 12, no. 1, pp. 36-48, Feb. 2003.

[28] T. Mckelvey, A. J. Fleming, and S. O. R. Moheimani, "Subspace-based system identification for an acoustic enclosure," Trans. ASME, J. Vibr. Acoust., vol. 124, no. 3, pp. 414-419, July 2002.

[29] T. McKelvey, H. Ackay, and L. Ljung, "Subspace-based identification of infinite-dimensional multi-variable systems from frequency-response data," IEEE Trans. Automat. Contr., vol. AC-41, pp. 960-979, 1996. 